(Aus dem physiologischen Institut der Universität Wien.)

\title{
Über die Widerstandsfähigkeit der Tenebriolarven gegen Austrocknung.
}

\author{
Von
}

stud. med. Bruno Berger.

Bekanntlich decken im allgemeinen die Lebewesen ihren Bedarf an Wasser, das als Lösungsmittel für die verschiedenen chemischen Umsetzungen erforderlich ist, durch direkte Wasseraufnahme. Der normale Ablauf der Lebensvorgänge ist an einen gewissen Durchfeuchtungsgrad der Gewebe gebunden, und eine Austrocknung, welche gewisse, meist eng gezogene Grenzen überschreitet, führt regelmässig den Tod herbei. Nur eine beschränkte Zahl von tierischen Organismen oder deren Keime, so insbesondere gewisse Rotatorien, Tardigraden und Nematoden, vermögen eine weitgehende Austrocknung lange zu überdauern, indem ihre Lebensvorgänge und ihr Stoffwechsel sozusagen latent werden, um bei Wasserzufuhr zu neuem Leben zu erwachen.

Um so bemerkenswerter erscheint das Vermögen gewisser Insekten, lange Zeit in einem relativ trockenen Medium zu einer normalen Entwicklung ihrer Lebensfunktionen zu gelangen; so z. B. die Fähigkeit der Larve der Pelzmotte, in sicherlich sehr wasserarmen Wollstoffen, Federn und Pelzen zu gedeihen, sowie auch die Existenz der sogenannten Mehlwürmer, d.h. der Larven des Käfers Tenebrio molitor in im gewöhnlichen Sinne trockenen Mehle. Es drängt sich die Frage auf, ob nicht die Existenzfähigkeit der genannten Lebewesen unter anscheinend so abnormen Bedingungen vielleicht darauf beruhe, dass denselben die Fähigkeit innewohnt, das für den normalen Ablauf der Lebensfunktionen selbstverständlich unentbehrliche Wasser aus der aufgenommenen trockenen Nahrung in ihrem Stoffwechsel selbst zu produzieren. Wissen wir doch, dass hungernde Tiere ihren Wasserbedarf grossenteils durch die Ver- 
brennung ibres eigenen Fettes decken. Falls also der Mehlwurm auf wasserfreiem Mehl wirklich zu gedeihen vermag, so könnte dies in dem Umstande begründet sein, dass er das Wasser nicht wie andere Tiere als solches aufnimmt, sondern mit jenem Wasser sein Auskommen findet, welches bei der Oxydation seiner Nahrung im Stoffwechsel entsteht. Wird ja doch z. B. die Stärke, die wohl ein Hauptnabrungsmittel dieser Larve bildet, im Organismus in letzter Linie zu Kohlensäure und Wasser verbrannt.

Von dieser Überlegung ausgehend, hat mich Herr Hofrat Sigmund Exner mit der Aufgabe betraut, zu untersuchen, wie sich die Tenebriolarven der Austrocknung gegenüber verhalten, ob sie befähigt sind, in einem völlig wasserfreien bzw. sehr wasserarmen Medium längere Zeit zu existieren oder gar zu wachsen, und wie der relative Wassergehalt ihrer Leibessubstanz unter den genannten Verbältnissen beschaffen ist.

Während über das Verhalten der Wirbeltiere bei Wasserentziehung mehrere Arbeiten erschienen sinà, ist mir keine Arbeit über das dieser niederen Tiere bei Wasserentziehung bekannt. Es ist festgestellt, dass hungernde Tiere länger leben als durstende und sogar oft die Wasseraufnahme verweigern. Durstende nehmen oft wenig Nahrung zu sich. Nothwang berichtet von durstenden Tauben, denen er Nahrung aufzwang ${ }^{1}$ ). Diese liessen grösstenteils die Nahrung im Kropfe zurück und deckten einen Teil des Wasserberlarfes durch Oxydation der Körpersubstanz. Nothwang berechnete, dass ungefähr die Hälfte des ausgeschiedenen Wassers durch Oxydation von Körpersubstanz entstanden sei. Nach $4^{1 / 2}$ Tagen starben die Tauben gewöhnlich. Pernice und Scagliosia) fanden, dass bei verdursteten Tieren sich eine allgemeine Zellatrophie finde. Dies stimmt mit den eben erörterten Erfahrungen überein. Durig stellte Versuche an Fröschen $a^{3}{ }^{3}$ ). Er stellte fest, dass der Organismus die wichtigeren Organe, wie Herz und Gehirn, auf Kosten minder wichtigerer Organe, wie Fett, Muskel, zu schützen

1) Nothwang, Die Folgen der Wasserentziehung. Arch. f. Hygiene Bd. 14. 1892.

2) Pernice und Scagliosi, Über die Wirkung von Wasserentziehung auf Tiere. Virchow's Arch. Bd. 139. 1895.

3) Durig, Wassergehalt und Organfunktion. Aus dem physiologischen Institut der Universität Wien. Pflüger's Arch. Bd, 85 u. 87. 1901. 
sucht. Dann bewies er, dass Frösche das Wasser beinahe gänzlich durch die Haut aufnehmen. Bei seinen Versuchen über die Wasseraufnahme durstender Tiere aus hyper- und hypisotonischen Lösungen zeigte es sich, dass die durstenden Frösche aus byperisotonischen Lösungen Wasser aufnahmen, wobei ein sehr grosser osmotischer Druck zu überwinden war.

Es sei mir nun gestattet, ubber meine Versuche in Kürze zu berichten:

1. Um zunächst das Verhalten der Tenebriolarven in einem absolut trockenen Medium festzustellen, wurde eine grössere Menge derselben $(93,33 \mathrm{~g}$ Gesamtgewicht) in einer Kristallisierschale, welche bei $105^{\circ}$ getrocknete Kleie enthielt, unter eine geräumige, auf eine Platte aufgeschliffene, tubulierte Glasglocke gebracht, durch welche mit Hilfe eines Aspirators täglich einige Zeit lang ein Strom durch konzentrierte Schwefelsäure getrockneter Luft geleitet wurde. Durch eine in der Glocke befindliche Schale mit konzentrierter Schwefelsäure war für die Absorption der von den Würmern abgegebenen Wassermengen gesorgt. In diesem absolut trocknen Medium sind einige wenige Würmer 4 Wochen lang an Leben geblieben, während die Mehrzahl der Larven durch Vertrocknung in der 3 . oder 4 . Woche zugrunde gegangen waren.

Bestimmungen je zweier Proben von Larven ergaben (die Trocknung der feingehackten Würmer bei $100^{\circ}$ bis zur Gewichtskonstanz erforderte 2-3 Wochen) zu Beginn des Versuches $38,44 \%$ und $38,39 \%$, im Mittel 38,41\% Trockensubstanz. Jene wenigen Würmer, welche die vierwöchentliche Trockenperiode überlebt haben, enthielten $35,04 \%$ Trockensubstanz, während Proben der Hauptmenge der abgestorbenen Würmer 52,98 \% und $52,46 \%$, durchschnittlich $52,72 \%$ Trockensubstanz enthielten. Aus dem Gesamtgewicht der Wurmmenge vor Beginn (93,33 g) und nach Beendigung des Versuches $(40,35 \mathrm{~g})$ ergibt sich auf Grund der mitgeteilten Prozentwerte, dass dieselben vor Beginn des Versuches $35,88 \mathrm{~g}$, nach Beendigung desselben aber nur mehr 26,02 g Trockeusubstanz enthalten haben. Die Wurmmasse hatte also infolge von. Dissimulationsprozessen (von Fäulnis war in dem trockenen Medium keine Spur zu bemerken) $9,86 \mathrm{~g}$ an trockener Leibessubstanz eingebüsst; das ist mehr als ein Viertel der ursprünglichen Körpersubstanz. Offenbar haben also von vornherein die Dissimulationsprozesse die Assimilationsvorgänge be- 
deutend übertroffen, und es ist überbaupt sebr fraglich, ob die Würmer unter diesen Verbältnissen Nahrung zu sich genommen laaben. Bemerkenswert erscheint die Tatsache, dass, während die abgestorbenen Larven einer schnellen postmortalen Austrocknung anheimfielen (Anstieg der Trockensubstanz auf ca. $52 \%$ ), die lebenden Tiere ihr Wasser selbst in diesem absolut trockenen Medium mit grosser Beharrlichkeit festhielten, derart, dass die nach überstandener vierwöchiger Trockenheitsperiode analysierten Individuen nicht nur nicht relativ wasserärmer, sondern sogar ein weniges wasserreicher erschienen als die normalen Larven. Die Differenz fällt in die Breite individueller Schwankungen, welche etwa zwischen $35-40 \%$ Trockensubstanz liegen.

II. Der Versuch wurde nunmehr mit einer abgekürzten Versuchsdauer von 17 Tagen wiederholt, welche sämtliche Larven überlebten. Eine geringe Abweichung lag in dem Umstande, dass in der ersten Versuchshälfte die Schwefelsäure durch Chlorcalcium ersetzt worden war. Proben der Larven wurden am 1., 10. und 17. Tage entnommen und analysiert. Es fand sich:

\begin{tabular}{c|c|c}
\hline Tag & $\begin{array}{c}\text { Trocken- } \\
\text { substanz } \\
0 / 0\end{array}$ & $\begin{array}{c}\text { Gewicht eines } \\
\text { Tieres durchschnittl. } \\
\text { g }\end{array}$ \\
\hline 1. & 35,9 & 0,150 \\
10. & 41,6 & 0,111 \\
17. & 41,6 & 0,118
\end{tabular}

Während die Würmer etwa ein Viertel ihres Körpergewichtes verloren haben, hat ihr prozentischer Wassergehalt, wenn überhaupt, so doch sicherlich nicht sehr wesentlich abgenommen, d. h. die Abnahme des Wassers und der Leibessubstanz haben offenbar miteinander annähernd gleichen Schritt gehalten.

III. In einem dritten Versuche sollte nun festgestellt werden, wie sich die Mehlwürmer unter Bedingungen verhalten, welche ihren natürlichen Lebensbedingungen nahe kommen. Sie wurden auf in gewöhnlichem Sinne trockenem Mehle, das jedoch noch nach der Analyse $12 \%$ Wasser enthielt, unter Zutritt von nicht getrockneter Luft gehalten. Je zwei in Intervallen von 3 Tagen entnommene Proben ( $a$ und b) von 200 Mehlwürmern orgaben an Trockensubstanz: 
Über die Widerstandsfähigkeit der Tenebriolarven gegen Austrocknung. 611

\begin{tabular}{c|c|c|c}
\hline \multirow{2}{*}{ Tag } & \multicolumn{2}{|c|}{ Trockensubstanz in \% } & $\begin{array}{c}\text { Durchschnitts- } \\
\text { wert } \\
\text { \% } \%\end{array}$ \\
\cline { 2 - 4 } & $\mathrm{a}$ & $\mathrm{b}$ & 39,9 \\
\hline 1. & 40,21 & 39,63 & 40,9 \\
4. & 40,20 & 41,69 & 40,8 \\
7. & 41,22 & 40,43 & 41,3 \\
10. & 41,08 & 41,63 & 42,1 \\
14. & 42,30 & 41,91 & 43,4 \\
17. & 43,51 & 43,24 & 41,8 \\
21. & 41,47 & 42,21 & 40,5 \\
24. & 40,55 & zerbrochen & 40,3 \\
27. & 40,30 & 42,39 & 42,1 \\
30. & 41,80 & &
\end{tabular}

Das durch die Wägung von je 200 abgezählten Würmern ermittelte Gewicht einer Larve betrug

$$
\begin{aligned}
& \text { am 1. Tage . . . } 0,09706 \mathrm{~g} \\
& \text { "4. " . . . . } 0,09932 \mathrm{~g} \\
& " 7 . \quad \text {. . . . } 0,09307 \mathrm{~g} \\
& " 10 . \quad \text {. . . } 0,09540 \mathrm{~g} \\
& n \text { 14. } \quad \text {. . . . } 0,09740 \mathrm{~g} \\
& " 17 . \quad \text {. . . . } 0,09172 \mathrm{~g} \\
& " 21 . \quad \text {. . . . } 0,09168 \mathrm{~g} \\
& \text { "24. } " \text {. . . . } 0,10516 \mathrm{~g} \\
& \text { "27. } " \text {. . . . } 0,10326 \mathrm{~g} \\
& \text { "30. } " \text {. . . . } 0,09442 \mathrm{~g}
\end{aligned}
$$

Es hatte also während dieser 30 tägigen Versuchsperiode keine ausserhalb der Versuchsfehler und individueller Schwankungen liegende Verschiebung des Durchschnittsgewichtes noch des Wassergehaltes stattgefunden, also weder Eintrocknung noch Wachstum.

Es liegt demnach keinerlei Anhaltspunkt für die Annahme vor, dass die Tenebriolarven eine biologische Ausnahme hinsichtlich ihres Wasserhaushaltes bilden und etwa das Vermögen besitzen, durch Produktion von Wasser auf dem Wege der Verbrennung aufgenommener Nahrung ihren Flüssigkeitsbedarf zu decken. Wachstumsvorgänge sind offenbar bei diesen wie bei anderen Tieren an die direkte Wasseraufnahme gebunden. Bemerkenswert erscheint aber die Tatsache, dass die Tenebriolarven offenbar infolge Anpassung an ihre natürlichen Existenzbedingungen wochenlang einen Aufenthalt in einem absolut trockenen Medium zu überdauern, und dabei trotz des grossen absoluten Wasserverlustes den relativen Feuchtigkeitsgehalt 
612 Bruno Berger: Über die Widerstandsfähigkeit der Tenebriolarven etc.

ilures Körpers annähernd konstant zu erhalten vermögen, indem dissimilatorische Gewebseinschmelzung und Wasserabgabe einander parallel gehen.

Will man die für die Tenebriolarven gefundene Tatsache, dass sie ihr Wasserbedürfnis durch Verbrennung der Nahrung nicht zu decken vermögen, verallgemeinern und auch für die Larven der Pelzmotte gelten lassen, so muss man dieser, nach den Erfahrungen des täglichen Lebens, doch die Fähigkeit zuschreiben, das in den lufttrockenen Substanzen ihrer Nahrung (Haare, Federn) befindliche Wasser zum Wachstum ihres Körpers zu verwerten bzw. mit demselben das Auslangen zu finden, im Gegensatz zu den Mehlwürmern. Denn Mottenlarven machen auch im verschlossenen Schrank ihre volle Entwicklung durch. 